\title{
Mobile Phone as an Everyday Life Style Object - The Move from »Plain old Mobile« to »Electronic Brain in your Pocket«
}

\author{
Daniela Stärke, Stefan Kramer, Bernd Eylert
}

Zusammenfassung

Seit der Einführung des Mobiltelefons hat sich seine Nutzung sehr stark verändert. Mittlerweile sind sie zu wichtigen Hilfsmitteln im täglichen Leben geworden, z. B. als Organizer oder Nachschlagewerk. Hier drängt sich nun die entscheidende Frage auf, inwieweit mobile Dienste Zugriff auf die Daten der Nutzer bekommen. Um dies, aber auch die Akzeptanz von verschiedenen mobilen Diensten zu erforschen, wurden im Rahmen dieses Papiers Umfragen in Berlin und Brandenburg durchgeführt. Ein weiterer Gegenstand der Umfrage war es, herauszufinden, ob die Nutzer bereit sind, private Daten Diensten zur Verfügung zu stellen, damit diese die Daten zu einer großen Menge vereinen, um so Vorschläge zu den Interessen der Nutzer machen zu können.
Abstract

Since the mobile phone was introduced to the market, its way of using changed rapidly. Nowadays it is an important tool, e.g. as organizer or knowledge base in people's every days life. The important question is now: In which way mobile services will gain influence on private data. For this purpose surveys were conducted in Berlin-Brandenburg to get a serious database and to figure out customer's wishes and expectations regarding these services. Another reason for this survey was to find out, whether people are willing to provide their private data to programs or services that combine different kinds of those data, to connect these independent data to a huge database, to make suggestions of people's interests.

\section{Einführung}

Die Entwicklung und das Design der Mobiltelefone in den letzten 10 Jahren haben sich von Funktionen wie der einfachen Telefonie und Kurznachrichten zu einem Multifunktionsgerät mit Kamera, Internetzugang, etc. gewandelt. Am Anfang waren Mobiltelefone große, schwere und sperrige Geräte, die eine ständige Erreichbarkeit fast ausschlossen. Niemand wollte noch eine zusätzliche Tasche für das Mobiltelefon mit sich umher tragen. Daher wurden die Handys mit der Zeit kleiner, leichter und kompakter. Heute ist ein gegenläufiger Trend zu beobachten. Die Endgeräte werden wieder größer, vor allem der Bildschirm, und auch der Funktionsumfang nimmt zu. Heute gibt es kaum noch Geräte ohne Kamera, MP3-Player und Internetfunktion. Die in der Lehrveranstaltung »Telekommunikation und Gesellschaft « des Masterstudiengangs Telematik herausgearbeiteten Thesen zur Entwicklung von Technologien lassen sich auch in diesem Zusammenhang wieder erkennen. Zuerst ist hier die These
»Technologie formt Technologie« zu nennen. Ohne die jetzige Generation von Mobilfunkgeräten würde es auch diese Vielzahl von Funktionen oder Diensten nicht geben. Des Weiteren hat sich nicht nur die Form und Funktionsweise verändert, sondern auch das Design und die Bedienung allgemein. Wo man sich früher mit einem extra Knopf durch das Menü klicken musste, wird heutzutage der Finger auf den Touchscreen gedrückt und das gewünschte Programm ausgeführt.

Wie bereits erwähnt wurde, sind Mobiltelefone mit immer mehr Funktionen ausgestattet. Ob diese auch vom Nutzer benötigt werden, soll nachfolgend anhand einer repräsentativen Befragung beleuchtet werden. Je nach Ergebnis kann eine weitere These bzw. ein Teil der folgenden These verwendet werden. Diese These besagt zweierlei, nämlich zum Ersten, dass »technologische Entwicklung akzeptiert wird, weil sie erfolgreich ist « und zum Zweiten »das an neue Technologie geglaubt wird, weil sie von der Gesellschaft akzeptiert wird $«$. 


\section{Stand der Technik}

\subsection{Geräte}

\subsubsection{Apple iPad}

Anfang 2010 stellte der Gründer von Apple, Steve Jobs, die neueste Entwicklung aus dem Hause Apple, das iPad, vor (Apple3 2010). Das iPad soll die Brücke zwischen einem Laptop und einem Smartphone schlagen. Laut Firmenangaben soll es das beste Erlebnis bzw. die beste Erfahrung sein, damit ins Internet zu gehen, Emails zu lesen oder Fotos und Filme anzusehen. Dabei soll das Produkt sich den Nutzerbedürfnissen anpassen und nicht der Nutzer dem Gerät. Durch die Multitouch-Technologie ist das iPad noch intuitiver zu bedienen, als das iPhone oder vergleichbare Geräte. Des Weiteren kann das iPad als Gerät zum Lesen von eBooks verwendet werden. Dabei soll sich die Leseerfahrung deutlich von den bestehenden eBook-Readern unterscheiden.

Neben der Möglichkeit seine Musik sofort im iTunes-Store zu kaufen, sowie zusätzliche Programme oder Spiele im App-Store, gibt es auch die Möglichkeit seine digitale Bibliothek im iBookstore zu erweitern. Schon zum Start des iPad gab es eine Vielzahl an zusätzlichen Applikationen, teils kostenfrei teils kostenpflichtig, welche direkt für das iPad entwickelt wurden bzw. in naher Zukunft noch angepasst werden. Generell können auch iPhone-Apps auf das Gerät geladen werden, nur die Auflösung bzw. die Ansicht kann sich dann unterscheiden. Mit Hilfe des Apple-SDK kann Software speziell für das iPad entwickelt werden. Dies gibt Entwicklern und Unternehmen die Möglichkeit zusätzliche Dienste anzubieten bzw. das iPad für Services zu nutzen, die jetzt noch nicht vorstellbar sind. Es wird eine ähnliche Entwicklung erwartet, wie sie das iPhone vorgemacht hat. Zur Markteinführung des iPhone gab es nur eine geringe Anzahl an Apps. Heute stehen dem (zahlenden) Kunden über 100.000 Apps zur Verfügung. Diese stammen aus den verschiedensten Bereichen, wie Spiele, News, Finanzen, Bildung, etc.

Der Inhalt (hier Content genannt) kann sehr visuell für das iPad aufbereitet werden und der Internet Browser oder die Applikation kann als Fenster ins Internet fungieren. Da ein Großteil der Menschen sehr visuell veranlagt ist, kann u. a.
Werbung noch spezieller platziert oder aufbereitet werden. Apple erhofft sich auch eine zusätzliche Kooperation mit Verlagen, um in Zukunft das iPad als digitale Zeitung/Zeitschrift verwenden zu können (Apple3 2010).

Trotz aller positiven Eigenschaften die das iPad hat, gibt es auch Kritikpunkte, welche schon kurz nach der Veröffentlichung des Gerätes aufgetreten sind. Zum Einen ist hier die nicht zufriedenstellende WLAN-Verbindung zu nennen. Ohne eine ausreichende Verbindung lassen sich viele Dienste und Funktionen schwer nutzen und somit ist der Mehrwert nicht mehr gegeben (Joos 2010). Zum Anderen ist das Betriebssystem des Gerätes nicht in der Lage mehrere Aufgaben gleichzeitig durchzuführen (Multitasking) (Fucci 2010). Des Weiteren wurde kritisiert, dass bei direkter Sonneneinstrahlung das Gerät seinen Betrieb einstellt, da es zu heiß geworden ist. Somit fällt eine Bedienung im Sommer im Freien nahezu aus. Ebenso kann die Bedienung durch die glänzende Oberfläche beeinträchtigt werden, da sich Lampen aller Art im Display spiegeln. Außerdem wird die Ausstattung an Software kritisiert. Dabei vor allem die fehlende Unterstützung des FlashPlayers von Adobe. Apple begründet dies damit, dass es Programmierfehler von Adobe gibt, die zu Systemabstürzen auf dem iPad führen könnten. Zudem würde die Akkulaufzeit erheblich negativ beeinträchtigt (chip1 2010).

\subsection{2 weTab}

Das weTab wurde von den deutschen Firmen neofonie GmbH und der 4tiitoo AG entwickelt und Anfang April 2010 vorgestellt (chip2 2010). Ursprünglich wurde das Gerät unter dem Namen WePad (WeTab2 2010) in Anlehnung an das Apple iPad bekannt gegeben und dann später umbenannt. Der Tablet-Computer funktioniert ähnlich wie sein amerikanischer Konkurrent, nur soll er in den am iPad kritisierten Punkten besser abschneiden. Das weTab hat die Möglichkeit Adobe Flash abzuspielen. Auch lassen sich mit Hilfe einer Webcam Videochats durchführen und das Gerät verfügt über 2 USB-Schnittstellen um zusätzliche Peripherie anzuschließen. Des Weiteren ist das weTab Betriebssystem multitaskingfähig, was das Arbeiten mit dem Gerät wesentlich vereinfacht (WeTab3 2010). 


\subsection{Applications - apps}

App ist die Kurzform für Applikation bzw. englisch application. Diese kleinen Programme funktionieren zumeist auf Smartphones und sind speziell für das entsprechende Gerät programmiert. Einer der größten Anbieter für Apps ist Apple mit seinem App-Store, in welchem Apps für das iPhone und jetzt auch für das iPad angeboten werden. Für das Google Smartphone mit dem Betriebssystem Android werden Apps im eigenen Adroid Market (wikipedia 2010) angeboten. Sogar Amazon will einen eigenen »Laden« für seinen eBookReader »Kindle« aufmachen (Lischka 2010). Apps sind für die verschiedensten Rubriken erhältlich, $u$. a. für Unterhaltung, Finanzen, soziale Netzwerke (wikipedia 2010), (Apple3 2010), (Android 2010).

Das Geschäftsmodell für die Applikationen funktioniert so, dass Entwickler ihre Software in das Angebot der verschiedenen App-Stores hinzufügen und nach eventueller Prüfung des Inhaltes, z. B. bei Apple, steht das Programm den (zahlenden) Kunden zur Verfügung. Wird das Programm heruntergeladen, erhält der Entwickler einen bestimmten Anteil (z. B. 70\%) des Preises. Der Rest wird vom Anbieter einbehalten. Apple führt eine Inhaltsprüfung der Apps durch und wenn das eingestellte Programm nicht den Anforderungen genügt, wird die App nicht freigegeben. Anders wird im Fall von Android verfahren. Dort wird eine Applikation ohne Prüfung zur Verfügung gestellt und wenn Beschwerden auftreten, wird entschieden, ob die Applikation von der Plattform genommen wird oder nicht. Daraus ergeben sich aber auch Probleme. Softwareentwickler sind nicht mehr so frei in ihrer Gestaltung bzw. in der Entwicklung generell. Ob eine Software erfolgreich ist und vom Nutzer angenommen wird, ist zum größten Teil vom Betreiber des App-Stores abhängig. Blockiert der Betreiber die Veröffentlichung auf seiner Plattform, hat der Entwickler keine legale Möglichkeit mehr seine Software für dieses bestimmte Gerät auf den Markt zu bringen. Dadurch kann Innovation gestoppt bzw. verlangsamt werden (Stöker 2010).

\subsection{Dienste}

\subsubsection{Mobile Commerce}

Mit der Entwicklung der Datenübertragung in Mobilfunknetzen und damit verbunden der Nutzung verschiedener Dienste des Internets auf mobilen Geräten stieg die Akzeptanz von Dienstleistungen aus dem Bereich des electronic Business. Um die Besonderheit der mobilen Datenübertragung hervorzuheben wurde nun vom mobile Business gesprochen. Aus diesem Begriff entstand in der Folgezeit der Begriff des »mobile Commerce «. Er ist in (Turowski/Pousttchi 2004) wie folgt charakterisiert: »Mobile Commerce bezeichnet jede Art von geschäftlicher Transaktion, bei der die Transaktionspartner im Rahmen von Leistungsanbahnung, Leistungsvereinbarung oder Leistungserbringung mobile elektronische Kommunikationstechniken (in Verbindung mit mobilen Endgräten) einsetzen.«

Der Erfolg des Mobile Commerce hängt nicht zuletzt von der hohen Akzeptanz der mobilen Datenübertragungstechniken und der zunehmenden Zahl der Funktionen der mobilen Geräte ab. Dank der mobilen Endgeräte ist es den Dienstanbietern nun möglich, den Nutzer zielgerichtet mit Informationen zu versorgen. Aus der Nutzung verschiedener OnlineDienste ist es den Providern nun möglich Statistiken zu führen, aus denen die Nutzung der verschiedenen Dienste durch den Nutzer hervorgeht. Die Auswertungen dieser Statistiken erlauben den Providern nun die Nutzer mit der Art von Informationen zu versorgen, die direkt auf sie zugeschnitten sind. Hieraus ergeben sich verschiedene Geschäftsmodelle von denen eine Auswahl im Folgenden näher betrachtet wird (Simonitsch 2003).

Content Provider: Sie entwickeln den Inhalt, der über die verschiedenen Vertriebskanäle an die Kunden weitergegeben wird. Auch sie haben zwei verschiedene Vertriebswege: den direkten oder den über das Portal (Huang et al. 2007).

Application Provider: Die Hauptaufgaben liegen bei der Aufbereitung des Inhalts bzw. der Anwendung sowie der Vertrieb. Dieser kann direkt erfolgen oder über ein Portal (vgl. App-Store), von dem der Kunde den Inhalt oder die Anwendung herunterladen kann. Die Application Provider sind hierbei nicht notwendigerweise mit den Firmen identisch, die den Inhalt entwickelt haben (Content Provider).

Für die Application Provider ergeben sich nun auch verschiedene Ertragsmodelle:

- direktes Modell

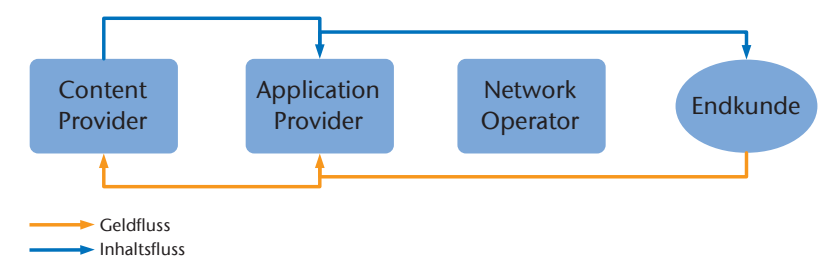

Abb. 1: direktes Modell, nach (Simonitsch, K. 2003) 
Aus Abbildung 1 kann man erkennen, dass bei diesem Modell der Inhalt vom Application Provider direkt an den Endkunden geliefert wird. Dieser zahlt dann für die Nutzung des Inhaltes auch wieder direkt an den Application Provider, der wiederum einen Teil an den Content Provider abführt.

- Provider Modell

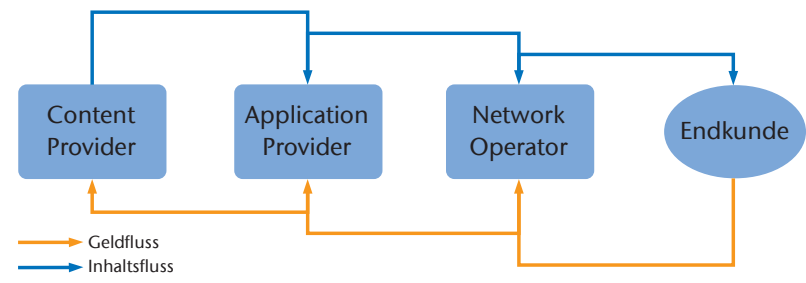

Abb. 2: Provider-Modell, nach (Simonitsch 2003)

Der Kunde bezieht den Inhalt in diesem Fall vom Network Operator und zahlt auch die Gebühren bei diesem. Der wiederum entrichtet einen Teil der Gebühren, die er vom Kunden eingenommen hat, an den Application Provider, der den Inhalt vorher von einem Content Provider bezogen hat.

- indirektes Modell

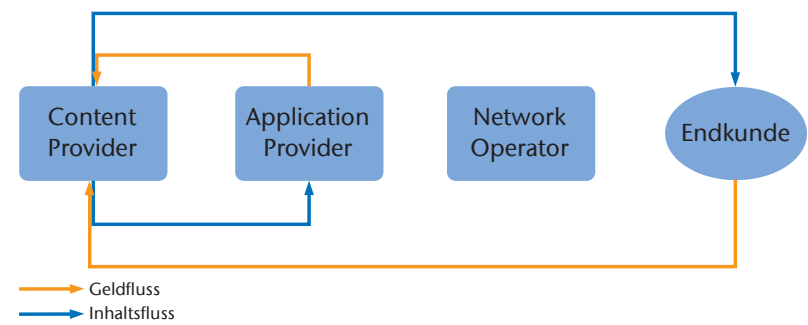

Abb. 3: indirektes Modell, nach Simonitsch (2003)

Anders als in den zuvor geschilderten Fällen verlaufen die Flüsse beim indirekten Modell. Hier stellt der Application Provider dem Content Provider eine Art Plattform zur Verfügung auf der er seinen Inhalt anbieten kann. Der Kunde bezieht diesen also indirekt über die Plattform vom »Hersteller «. An diesen entrichtet er auch seine Gebühren. Der Content Provider seinerseits zahlt dem Application Provider eine Gebühr für die Nutzung der Plattform.

Virtual Operator: Als virtueller Operator wird ein Netzoperator bezeichnet, der kein eigenes Mobilfunknetz unterhält sondern dieses von anderen »realen« Netzoperatoren mit nutzt und dafür eine Gebühr entrichtet. Diese virtuellen Netzoperatoren haben den Vorteil, dass sie keine Kosten im Bereich der Unterhaltung eines eigenen Netzes haben und deshalb einen großen Teil der Kosten sparen und so sehr preisgünstige Angebote für den Kunden machen können.

\subsubsection{Location Based Services}

Die Idee von Location Based Services ist, dem Nutzer in Abhängigkeit von seinem aktuellen Standort mit Daten zu versorgen. In der Anfangszeit dieser Dienste sahen diese so aus, dass man zwischen Push- und Pull-Diensten unterschied (Penders 2004). Mittlerweile ist die Zahl der Smartphones stark angestiegen und damit auch die Zahl derer, die die Anwendungen (Apps) auf diesen Geräten nutzen. Eine Teilmenge dieser Apps sind auch solche, die auf die Positionsdaten des Nutzers zugreifen, z. B. die Gelbe-Seiten App fürs iPhone[1]. Da diese Geräte meist mit einem GPS Sensor ausgestattet sind, fällt die Positionsbestimmung dementsprechend leicht. Die folgende Abbildung zeigt schematisch den Informationsverlauf bei einer solchen Anwendung:

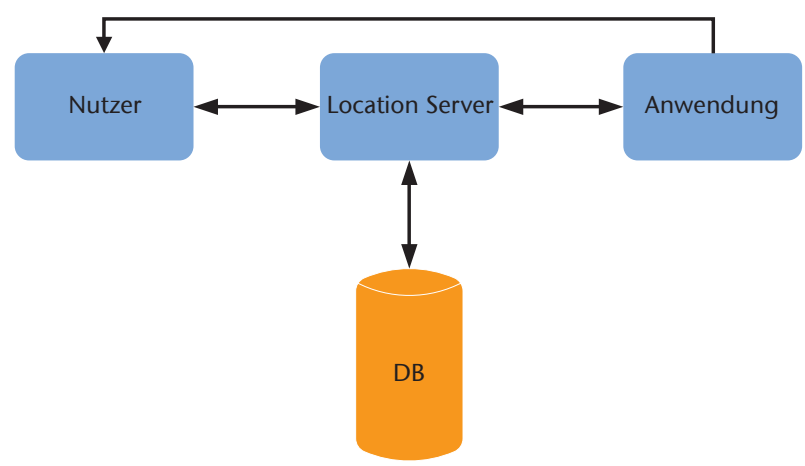

Abb. 4: schematische Darstellung von Location Based Services, nach Sun (2009)

Die Positionsdaten, die vom GPS Empfänger gelesen werden, werden über das Mobilfunknetz an einen Server übertragen, der die Daten in einer Datenbank (DB) ablegt. Auf diese Datenbank kann dann eine Anwendung, die Positionsdaten benötigt, zugreifen und diese dort abfragen. Der Nutzer erhält dann, von der Anwendung ausgehend, zu seinen Positionsdaten die gewünschten Informationen. Nach diesem Prinzip arbeitet die bereits erwähnte Gelbe-Seiten App auf dem iPhone.

Dies ist aber nicht das einzige Anwendungsfeld für Location-Based-Services. Man kann z. B. auch verschiedene Augmented-Reality-Anwendungen mit diesen Daten betreiben (Vaughan-Nichols 2009). So kann man beispielsweise über die Geodaten, die man vom GPS Empfänger des mobilen Geräts liest, und den Aufnahmen, die die Kamera des Geräts macht, erkennen, in welcher Umgebung sich der Nutzer befindet. Auf diese Weise kann man sehr viel genauere Informationen über die Gegend bekommen, als man sie nur mit den Geodaten hätte. Hat das System nun die Richtung erkannt, in 
die der Nutzer sieht, kann man ihn über das Mobilgerät mit Informationen versorgen. Steht man z. B. in einem Gebirge und hält das Gerät auf einen Berg in der Umgebung kann die Software Informationen wie Name, Höhe, Erstbestieg, etc. zu diesem Berg liefern.

In die gleiche Richtung gehen mobile Touristenführungssysteme, die den Touristen in Abhängigkeit von ihrem Aufenthaltsort Informationen zu bestimmten Sehenswürdigkeiten geben. Dieser Ansatz wurde in (Bao 2009) verfolgt, allerdings wurden hier neben den touristischen Informationen zusätzlich Multimediadaten an den Nutzer übertragen.

\section{Nutzerbefragung}

\subsection{Methoden}

Um ein repräsentatives Meinungsbild in Bezug auf die Bereitschaft, neuartige Dienste und Möglichkeiten von Mobiltelefonen zu erhalten, ist eine Befragung durchgeführt worden, bei der sowohl allgemeine Themen wie die Nutzung des Mobiltelefons als auch brisante, wie der Datenschutz, Gegenstand waren. Bei der Konzipierung des Fragebogens lag das Hauptaugenmerk darauf, keine Suggestivfragen zu stellen, damit die Befragten bei der Beantwortung nicht in eine bestimmte Richtung gedrängt werden. Stattdessen wurde versucht die Fragen so stellen, dass das Ergebnis das reale Meinungsbild möglichst genau widerspiegelt. Weiterhin musste bedacht werden, dass die Fragen so gestellt sind, dass sie binnen weniger als einer Minute verstanden werden und auf umfangreiche Erklärungen verzichtet werden kann, um so zu vermeiden, dass die befragte Person zu schnell das Interesse verliert.

Der so aufgestellte Fragebogen enthält nach diesen Kriterien 5 fachliche und 2 demografische Fragen, nach Altersgruppe und Geschlecht, die im Anhang aufgeführt sind.

\subsection{Hypothesen}

Aus den im Anhang vorgestellten Fragen ergeben sich für die Beantwortung dieser nun Hypothesen, die im Wesentlichen die Erwartungshaltung der Autoren an die Ergebnisse wiedergibt:

These 1: Die Mehrheit der Befragten nutzt das Mobiltelefon für die »klassischen« Dienste Telefonie und SMS. Das Mobiltelefon wird dabei auch für Multimediafunktionen wie Radio oder als MP3-Player verwendet.
These 2: Die Nutzer von mobilen Internetdiensten verwenden diese hauptsächlich für Emails oder diverse Informationsdienste.

These 3: Der Anteil der Nutzer von Spielen oder sozialen Netzwerken wird bei denen, die jünger als 40 Jahre sind deutlich höher ausfallen, als bei den anderen Altersgruppen.

These 4: Auf die Frage, ob Befragte eher ein einfaches Handy oder ein Smartphone kaufen würden, wird vermutet, dass doch eher einfache Telefone präferiert werden.

These 5: Die Bereitschaft der Befragten ihr Mobiltelefon für Finanztransaktionen zu verwenden ist gering.

These 6: Um die Bekanntheit von diversen Diensten zu steigern, sind die Nutzer wenig bereit private Daten wie Adressbücher zur Verfügung zu stellen.

\subsection{Datenauswertung}

Insgesamt wurden 52 Personen befragt, von denen 29 männlich und 23 weiblich waren. Dabei wurde darauf geachtet, dass sich die Befragung auch über alle relevanten Altersgruppen erstreckt. Eine detaillierte Auswertung der einzelnen Fragen ist in Anhang enthalten. Bei genauerer Betrachtung der Ergebnisse kann man feststellen, dass die Behauptungen, die in den Thesen aufgestellt wurden, mehrheitlich eingetreten sind. Die folgende Abbildung zeigt die aktuelle Nutzung des Mobiltelefons:

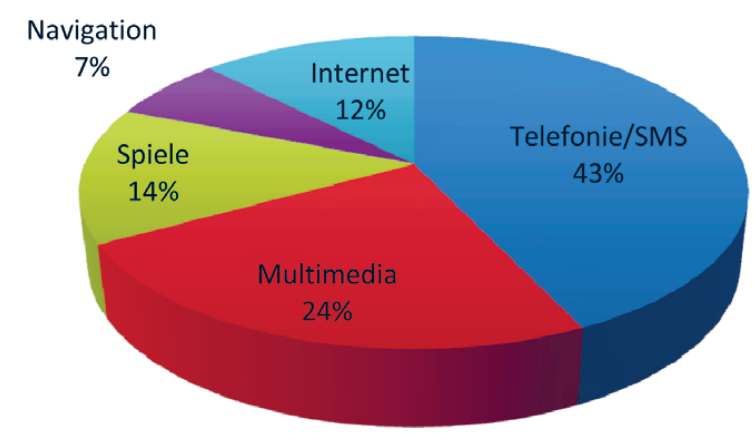

Abb. 5: Übersicht der verwendeten Dienste und Funktionen eines Mobiltelefons

Man kann hier sehr eindeutig sehen, dass das Mobiltelefon zurzeit noch am meisten für die »klassischen« Dienste Telefonie und SMS genutzt wird. Dienste wie Navigation oder mobiles Internet aber sehr stark auf dem Vormarsch sind, sodass man an diesem Punkt eine gewisse Entwicklung hin zur Akzeptanz und Nutzung multifunktionaler Geräte beobachten kann. 
Des Weiteren kann man aus den Ergebnissen der Befragung ableiten, dass die Nutzung von mobilen Internetdiensten ansteigt, wie man folgender Abbildung entnehmen kann.

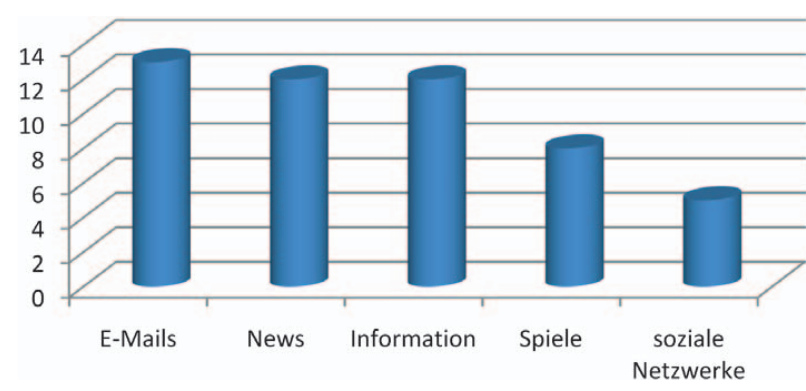

Abb. 6: Verwendung des mobilen Internets (Ergebnisse bezüglich der privaten Daten)

Die Ergebnisse dieser Frage zeigen, dass auch in diesem Feld die klassischen Dienste Email, News und Information bestimmend sind. Die relativ geringe Antwortzahl bei den sozialen Netzwerken lässt sich unter anderem dadurch erklären, dass diese vorwiegend von jungen Menschen genutzt werden, die aber, um eine gewisse Ausgeglichenheit zwischen den Altersgruppen zu erreichen, nicht ausschließlich befragt wurden.

Waren die Ergebnisse bei diesen beiden Fragen noch sehr ausgeglichen, so sind sie bei den folgenden beiden sehr eindeutig. Hauptgegenstand dieser Fragen ist der Datenschutz, die Ergebnisse stellen sich wie folgt dar:

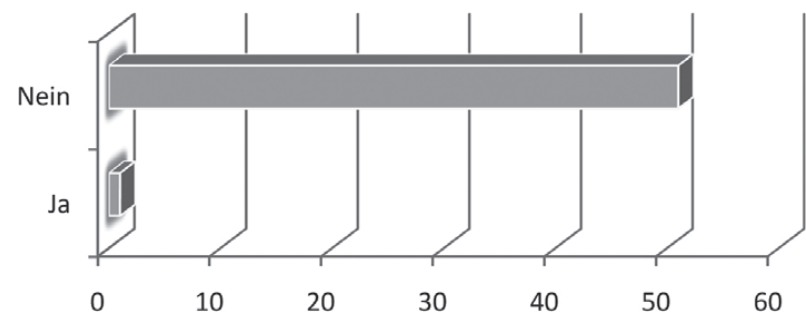

Abb. 7a: Bereitschaft, private Daten freizugeben (prozentuale Ergebnisse bezüglich der privaten Daten)

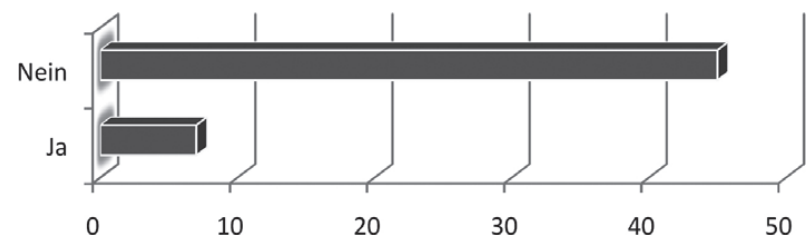

Abb. 7b: Bereitschaft, das Handy für Finanztransaktionen zu nutzen (prozentuale Ergebnisse bezüglich der privaten Daten)

\section{Ausblick}

In der vorliegenden Ausarbeitung wurde die Akzeptanz von multifunktionalen Mobiltelefonen und neuen Diensten des mobilen Internet untersucht. Es ist hierbei zu beobachten, dass sich die Zahl der Menschen, die sich für ein klassisches Mobiltelefon entscheiden würden und die derer, die sich ein für ein Smartphone entscheiden würden, nahezu ausgeglichen ist. Aus diesem Punkt kann man ableiten, dass die »neue « Generation von Geräten nicht bei allen Menschen gut ankommt, was zum einen an der ungewohnten Bedienung via Touchscreen liegen kann, zum anderen aber auch daran, dass man denken kann, ein solches Gerät wäre viel zu kompliziert und böte ohnehin Funktionen, die man nie bräuchte.

Aus der Weiterentwicklung der Geräte kann man auch die Weiterentwicklung von verschiedenen Diensten ableiten, was wiederum auf die eingangs erwähnte These »Technologie formt Technologie« zurück geführt werden kann. In diesem Fall ist aber nicht eindeutig zu identifizieren, welche Technologie nun welche geformt hat, da hier in vielen Fällen eine Symbiose herrscht, die nur schwer aufgelöst werden kann, da bestimmte Dienste eine gewisse Hardwareausstattung voraussetzen. Ab diesem Punkt kann man davon ausgehen, dass die Entwicklung der Hardware in diesem Bereich auch die Entwicklung der Dienste beeinflusst. Allerdings muss man bei den Diensten zwischen solchen die von den Nutzern akzeptiert werden, weil sie erfolgreich sind unterscheiden und solchen, bei denen an die Entwicklung geglaubt wird, weil sie von der Gesellschaft akzeptiert sind. Dieser Rückschluss auf die, auch in der Einführung erwähnte, These lässt sich auch und gerade durch die Ergebnisse aus der Befragung der Nutzer ableiten. Zu den Diensten, die akzeptiert werden, weil sie erfolgreich sind, zählt z. B. das mobile Internet. Es ist allgemein bekannt, was das Internet ist und welche Möglichkeiten es bietet. Also ist es nur logisch, wenn dieser Dienst auch auf mobilen Geräten angeboten wird. Es ist in jedem Fall zu beobachten, dass das mobile Internet genauso erfolgreich ist, wie das »klassische« vom PC aus. Es bleibt abzuwarten, ob es Dienste geben wird, die erfolgreich sind, weil sie von der Gesellschaft akzeptiert werden. In dieser Ausarbeitung wurde versucht, sich dem Thema »mobile Banking « in diesem Zusammenhang zu widmen. Es zeigte sich aber, dass im Moment noch eine sehr große Skepsis gegenüber diesem Thema bei den Menschen vorhanden ist. Um diese 
Skepsis in Zukunft in Akzeptanz zu verwandeln, bedarf es allerdings einiger Maßnahmen der Provider und Dienstleister (z. B. der Banken) wie der Schaffung zuverlässiger, verschlüsselter Verbindungen oder eindeutiger Bekenntnisse bzw. Maßnahmen den vertrauenswürdigen Umgang mit den Daten der Nutzer betreffend. Solange man solche sicherheitskritischen Vorgänge mit simplen Verfahren wie Phishing kompromittieren kann, ist es fragwürdig, ob die Akzeptanz eines solchen Dienstes in Zukunft steigen wird.

\section{Anmerkung}

[1] http://itunes.apple.com/de/app/gelbe-seiten/id312387605?mt=8

\section{Anhang: Fragebogen}

1) Welche Dienste/Funktionen nutzen Sie auf ihrem Mobiltelefon?

2) $\mathrm{Zu}$ welchem Zweck wird mobiles Internet verwendet?

3) Mobiltelefone werden immer umfangreicher an Funktionen. Wenn Sie die Wahl zwischen einem Handy, welches Telefonie und SMS ermöglicht und einem Gerät, welches wesentlich mehr Funktionen als ein gewöhnliches Mobiltelefon bereitstellt hätten, für welches würden Sie sich entscheiden?

4) Wären Sie bereit ihr Handy für Finanztransaktionen (z. B. als virtuelle Kreditkarte) zu verwenden?

5) Um die Bekanntheit von Diensten zu steigern, wären Sie bereit Daten aus Ihren persönlichen Adressbüchern den Diensten zur Verfügung zu stellen?

6) Angabe des Geschlechts

7) Zugehörigkeit zu Altersgruppe

\section{Literaturverzeichnis}

Android (2010): http://www.android.com/market/free.html, Zugriff 10.05.2010.

Apple1 (2010): http://www.apple.com/de/iphone/apps-for-iphone/, Zugriff 10.05.2010.

Apple1 (2010): Apple iPad - Demo Video, http://www.youtube.com/ watch?v=9q1RithxrzY, Zugriff 07.05.2010.

Apple2 (2010): Apple iPad - View photos and images, http://www. apple.com/ipad/gallery/, Zugriff 17.05.2010.

Apple3 (2010): Apple launches iPad - Magical \& Revolutionary Device at an Unbelievable Price. http://www.apple.com/pr/ library/2010/01/27ipad.html, Zugriff 07.05.2010.

Bao, X. et al. (2009): Integration of multimedia and Location Based Services on Mobile Phone Tour Guide System. In: Proceedings of the IEEE International Conference on Network Infrastructure and Digital Content, 642 - 646 . chip1 (2010): Apple: Steve Jobs über Flash beim iPad und Google, chip-online, http://business.chip.de/news/Apple-Steve-Jobsueber-Flash-beim-iPad-und-Google_41205502.html, Zugriff 09.05.2010.

chip2 (2010): WePad: Deutscher iPad-Konkurrent kommt im Juni, chip-online, http://www.chip.de/news/WePad-Deutscher-iPadKonkurrent-kommt-im-Juni_42024426.html, Zugriff 10.05.2010.

Fucci, A. (2010): 8 Things That Suck About the iPad. http://gizmodo.com/5458382/8-things-that-suck-about-the-ipad, Zugriff 10.05.2010.

Huang, W., Qi, L.Y., Dong, L.H. (2007): Business Models and Implementations of M-Commerce. In: Proceedings of the International Conference on Communications, Networking and Mobile Computing, $3637-3640$.

Joos, R. (2010): iPad-Überhitzung und WLan-Probleme: Erster TabletÄrger, http://www.macnotes.de/2010/04/06/ipad-uberhitzungund-wlan-probleme-erster-tablet-arger/, Zugriff 10.05.2010.

Lischka, K. (2010): Kindle-Content: Amazon baut App Store, http:// www.spiegel.de/netzwelt/gadgets/0,1518,673122,00.html, Zugriff 10.05.2010.

Penders, J. (2004): Privacy in (mobile) Telecommunications Services. Ethics and Information Technology, 6: 247 - 260.

Simonitsch, K. (2003): Mobile Business: Geschäftsmodelle, Kooperationen und Möglichkeiten zur Beurteilung und Analyse. Doktorarbeit, Karl-Franzens-Universität Graz.

Stöker, C. (2010): Smartphones, Kindle \& Co. Zwangsjacke für das Internet, http://www.spiegel.de/netzwelt/netzpolitik/0,1518,673182,00.html, Zugriff 10.05.2010.

Sun, Y. et al. (2009): A Flexible Privacy-Enhanced Location-Based Services System Framework and Practice. IEEE Transactions on mobile computing, 8(3):304 - 321.

Turowski, K., Pousttchi, K. (2004): Mobile Commerce, Springer-Verlag, Heidelberg.

Vaughan-Nichols, S.J. (2009): Augmented Reality: No Longer a Novelty?, Computer 42(12): $19-22$.

WeTab1 (2010): In Aktion - die Galerie - weTab, http://wetab.mobi/ galerie, Zugriff 17.05.2010.

WeTab2 (2010): Pressemitteilung zur Umbenennung 07. Mai 2010. http://wetab.mobi/pm-2010-05-07, Zugriff 10.05.2010.

WeTab3 (2010): Produktdetails, http://wetab.mobi/produktdetails, Zugriff 10.05.2010.

wikipedia (2010): wikipedia, http://de.wikipedia.org/wiki/App, Zugriff 10.05.2010.

\section{Autoren}

Daniela Stärke, B. Eng.

Telematik

Technische Hochschule Wildau [FH]

daniela.staerke@th-wildau.de

Stefan Kramer, B. Eng.

Telematik

Technische Hochschule Wildau [FH]

stefan.kramer@th-wildau.de

Prof. Dr. Bernd Eylert

Technische Hochschule Wildau [FH]

bernhard.eylert@th-wildau.de 